\title{
Myeloid dendritic cells induce $T h 2$ responses to inhaled antigen, leading to eosinophilic airway inflammation
}

\author{
Bart N. Lambrecht, ${ }^{1,2}$ Marijke De Veerman, ${ }^{3}$ Anthony J. Coyle, ${ }^{4}$ \\ Jose-Carlos Gutierrez-Ramos, ${ }^{4}$ Kris Thielemans, ${ }^{3}$ and Romain A. Pauwels ${ }^{2}$
}

${ }^{1}$ Department of Pulmonary Medicine, Erasmus University Rotterdam, Rotterdam, The Netherlands
2Department of Respiratory Diseases, University Hospital Ghent, Ghent, Belgium
${ }^{3}$ Department of Physiology, Free University Brussels, Jette, Belgium
${ }^{4}$ Department of Biology, Inflammation Division, Millennium Pharmaceuticals, Cambridge, Massachusetts, USA

Address correspondence to: Bart N. Lambrecht, Department of Pulmonary Medicine, Erasmus University Rotterdam, Room Ee2263, Dr. Molewaterplein 50, 3015 GE Rotterdam, The Netherlands.

Phone: 31-10-4087703; Fax: 31-10-4089453; E-mail: lambrecht@lond.azr.nl.

Received for publication August 10, 1999, and accepted in revised form June 7, 2000.

\begin{abstract}
The aim of this study was to investigate whether dendritic cells (DCs) can induce sensitization to aeroallergen in a mouse model of allergic asthma. Ovalbumin-pulsed (OVA-pulsed) or unpulsed myeloid DCs that were injected into the airways of naive mice migrated into the mediastinal lymph nodes. When challenged 2 weeks later with an aerosol of OVA, activated CD4 and CD8 lymphocytes, eosinophils, and neutrophils were recruited to the lungs of actively immunized mice. These CD4 $4^{+}$lymphocytes produced predominantly IL- 4 and IL- 5 but also IFN- $\gamma$, whereas CD8 $8^{+}$lymphocytes produced predominantly IFN- $\gamma$. Histological analysis revealed perivascular and peribronchial eosinophilic infiltrates and goblet cell hyperplasia. Studies in $\mathrm{IL}_{-4^{-/-}}$and $\mathrm{CD} 28^{-/-}$mice revealed that production of IL-4 by host cells and provision of costimulation to T cells by DCs were critical for inducing the response. Lung $\mathrm{CD}^{+} \mathrm{T}$ cells strongly expressed the Th2 marker $\mathrm{T} 1 / \mathrm{ST} 2$, and signaling through this molecule via a ligand expressed on DCs was essential for the establishment of airway eosinophilia. These data demonstrate that DCs in the airways induce sensitization to inhaled antigen and that molecules expressed on the surface of these cells are critical for the development of Th2-dependent airway eosinophilia.
\end{abstract}

J. Clin. Invest. 106:551-559 (2000).

\section{Introduction}

Allergic asthma is now regarded as a $\mathrm{T}$ cell-mediated disorder in which sensitized individuals develop eosinophilic airway inflammation in response to inhalation of aeroallergen (1). T lymphocytes respond to antigen after it has been presented as immunogenic peptides in the context of MHC molecules. Naive T cells proliferate and differentiate in response to T-cell receptor (TCR) recognition of peptide-MHC only when accompanied by costimulatory signals (e.g., CD80 and CD86) provided on the surface of professional APCs. It is now clear that the dendritic cell (DC) is the most powerful antigen-presenting cell (APC) for inducing a primary immune response in vivo. It has potent antigen capturing and presenting capacity and readily expresses costimulatory ligands for naive T cells (2). The DC network within the epithelium of the conducting airways is ideally positioned to perform a surveillance role for inhaled antigens/allergens, and as the only resident cell population expressing surface MHC class II in normal (noninflamed) epithelium, the DCs are likely to play an important role in primary allergic sensitization to aeroallergens (3-5). After antigen uptake, airway DCs migrate to the paracortical T-cell zone of the draining lymph nodes (LNs) of the lung, where they interact with naive $T$ cells (6). During this interaction with naive $T$ cells, the DC becomes a reporter of its earlier microenvironment and has the potential to induce a polarized Th1 or Th2 type of response (7). The determining factors by which DCs will induce either a Th1 or a Th2 type of response are multiple and relate to the type of DC presenting the antigen $(\mathrm{Ag})(8,9)$, their capacity to produce polarizing cytokines such as IL-12 and IL-6 (10), the maturity of the DC network (11), the degree and type of costimulation provided to T cells (12), and the presence of inflammation during Ag encounter (11).

Despite the critical role that DCs might play in the polarization of Th responses in the airways, few studies have examined the contribution of DC to Th2-driven sensitization to aeroallergen. The purpose of this study was to investigate whether and under which conditions injection of Ag-pulsed DCs into the airways leads to sensitization to inhaled Ag and to the development of Ag-induced airway eosinophilia.

\section{Methods}

Experimental animals. C57BL/6 mice were purchased from Harlan (Zeist, The Netherlands) and housed 
under specific pathogen-free conditions in sterile microisolator units. C57BL/6 mice with a targeted mutation of the IL-4 gene $\left(I L 4^{t m 1 C g n}\right)$, with a targeted mutation of the CD28 gene $\left(C D 28^{-/-}\right)$, and control wild-type littermates were purchased from The Jackson Laboratory (Bar Harbor, Maine, USA).

Culture and antigen loading of bone marrow DCs. DCs were isolated as reported in detail previously $(13,14)$. Bone marrow was magnetically depleted of $\mathrm{CD}^{+}$, $\mathrm{CD}^{+}, \mathrm{MHC}$ class $\mathrm{II}^{+}, \mathrm{B} 220^{+}$and $\mathrm{Gr}-1^{+}$cells. Lineage ${ }^{-}$ cells were grown in medium supplemented with 10 $\mathrm{ng} / \mathrm{mL}$ of rhFlt3-ligand, $200 \mathrm{ng} / \mathrm{mL}$ rmGM-CSF and 1 $\times 10^{3} \mathrm{U} / \mathrm{mL} \mathrm{rmIL-4}$. For transfer experiments into IL4-deficient animals, DCs were grown in the absence of exogenous IL-4. After 4 days of culture, cells were placed in serum-free medium (Optimem-1; N.V. Life Technologies SA, Merelbeke, Belgium). At day 9, nonadherent cells were collected and enriched by density centrifugation over metrizamide (14.5\% wt/vol; $600 \mathrm{~g}$ for 20 minutes; Sigma Chemical Co., St. Louis, Missouri, USA). After purification, FACS analysis (Becton Dickinson Immunocytometry Systems, San Jose, California, USA) revealed that almost all cells coexpressed MHC class II and the DC marker CD11c; greater than 95\% expressed the costimulatory molecules CD86, HSA, CD44, and ICAM-1, and approximately 50\% expressed CD80 as reported in detail previously (14).

In initial experiments, purified DCs were incubated for 2 hours with $100 \mu \mathrm{g} / \mathrm{mL}$ of ovalbumin (OVA) (grade V; Sigma Chemical Co.) or, as a control, with medium alone, followed by extensive washing to remove free OVA. In later experiments, the OVA-pulsing period was performed overnight, leading to enhanced immunostimulatory capacity (see later here).

Intratracheal injection of DCs. Mice were anesthetized (avertin 2.5\% wt/vol in PBS), and $80 \mu \mathrm{L}$ of cell suspension was injected intratracheally, using the technique of Ho and Furst (15). To study the distribution of injected DCs, cells were labeled with the green fluorescent dye carboxyfluorescein diacetate succinimidylester (CFSE; Molecular Probes Inc., Eugene, Oregon, USA), essentially as described elsewhere (6). A total of $1 \times 10^{6}$ DCs were instilled intratracheally into naive mice and traced 12 hours, 36 hours, and 120 hours later in the bronchoalveolar lavage fluid (BALF), lung tissue, and mediastinal and inguinal LNs. Lungs were first perfused via the right ventricle using PBS, followed by BAL (see later here). Excised tissues were minced, incubated for 90 minutes in $100 \mathrm{U} / \mathrm{mL}$ collagenase IV (N.V. Life Technologies SA) and $0.02 \mathrm{mg} / \mathrm{mL}$ DNaseI (Sigma Chemical Co.), and $\mathrm{CFSE}^{+} \mathrm{CD} 11 \mathrm{c}^{+} \mathrm{DC}$ were enumerated using flow cytometry.

Immunization with antigen-pulsed DCs and secondary exposure to antigen aerosol. On day 0 , groups of mice ( $n$ $=6-8$ per group) were immunized by intratracheal instillation of either $1 \times 10^{6}, 1 \times 10^{5}$, or $1 \times 10^{4}$ OVADCs. As a control, a group of mice received $1 \times 10^{6}$ control PBS-DCs. From day 14 to day 20, mice were exposed either to seven OVA aerosols $(10 \mathrm{mg} / \mathrm{mL}$; grade III in PBS) or seven PBS aerosols, on consecutive days for 30 minutes daily (5).

Treatment with $m A b$ 's. To study the effect on sensitization of blocking the interaction of T1/ST2 with its ligand, $50 \mu \mathrm{g}$ of purified rat mAb 3E10, recognizing the T1/ST2 molecule on Th2 cells (16) was injected intratracheally simultaneously with OVA-DCs at day 0 . Rat IgG1 was used as a control. In separate experiments, mice received $30 \mu \mathrm{g}$ of T1/ST2-Ig fusion protein that directly competes with membrane-bound T1/ST2 for binding with its putative ligand and hence blocks T1/ST2 signaling (16). Human Ig (hu-Ig) was used as control.

$B A L$. Twenty-four hours after the last aerosol exposure, mice were killed and BAL was performed (5). The BALF was centrifuged, cells were counted, and supernatant was stored. Differential cell counts were performed on cytospin preparations stained with MayGrünwald-Giemsa. Aliquots of $2 \times 10^{5}$ cells were prepared for flow cytometry.

Flow cytometry. The following antibodies were used to stain cells obtained from BALF and/or LNs: CD3EPECy5, CD4-FITC, CD8 $\alpha$-PE, CD25-PE, CD69-PE, T1/ST2 (3E10, rat IgG1). On bone marrow DC prepa-

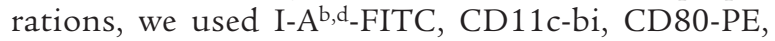
CD86-PE, CD44-PE, HSA-PE, ICAM-1-PE, and B-cell mAb CD45R-PE (B220). Antibodies were obtained commercially from PharMingen (San Diego, California, USA) or were from American Type Culture Collection (Manassas, Virginia, USA). Biotinylated antibodies were detected using streptavidin-PECy5 (Quantum Red; Sigma Chemical Co.). Unconjugated rat $\mathrm{mAb}$ 's were detected by secondary goat anti-rat IgG-PE (Jackson Immunoresearch Laboratories, West Grove, Pennsylvania, USA). Expression of the T1/ST2ligand on DC was demonstrated by binding of T1/ST2-Ig fusion protein, followed by anti-human IgG1-FITC (16). Samples were analyzed on a FACSVantage flow cytometer using Cell Quest software (Becton Dickinson Immunocytometry Systems).

For the single-cell detection of intracellular cytokines, BALF cells were stimulated for 5 hours at $3 \times 10^{6}$ cells/ $\mathrm{mL}$ in plates coated with anti-CD3 antibody $(2$ $\mu \mathrm{g} / \mathrm{mL}$ of $145-2 \mathrm{C} 11$ antibody), in the presence of antiCD28 mAb to provide costimulation, or with PMA/ionomycin (17). After 2 hours, monensin $(2 \mu \mathrm{M}$; PharMingen) was added. After washing, cells were incubated with $2.4 \mathrm{G} 2$, followed by surface staining for CD3PECy5 and CD4-FITC. Fixation, permeabilization, and staining for intracellular cytokines was performed using anti-IFN- $\gamma$-allophycocyanin (XMG1.2), anti-IL-4-PE (11B11), and anti-IL-5-allophycocyanin (TRFK-5) mAb's, according to PharMingen's protocol. Samples were analyzed on a FACScalibur flow cytometer (Becton Dickinson Immunocytometry Systems).

Cytokine measurements. The cytokine content in unconcentrated BALF was determined using ELISA (IFN- $\gamma$ Quantikine M; R\&D Systems, Abingdon, United Kingdom; IL-4 from Biotrak, Life Science, Amer- 


\section{Figure 1}

(a) Localization of $\mathrm{CFSE}^{+}$DC 36 hours after intratracheal injection of $1 \times 10^{6} \mathrm{DC}$ s into naive mice. DC were identified in BALF, digested lung tissue, and mediastinal and inguinal LNs as CD3-B220- cells of low autofluorescence, strongly expressing CD11c. Injected DCs can be discriminated from endogenous DCs by green fluorescence of CFSE. The percentage of CFSE ${ }^{+}$DCs among all LN cells is indicated. (b) Kinetics of appearance of CFSE ${ }^{+}$DC in BALF (open squares, left $y$ axis) and mediastinal LNs (filled squares, right $y$ axis). Representative of five mice per time point in the group.

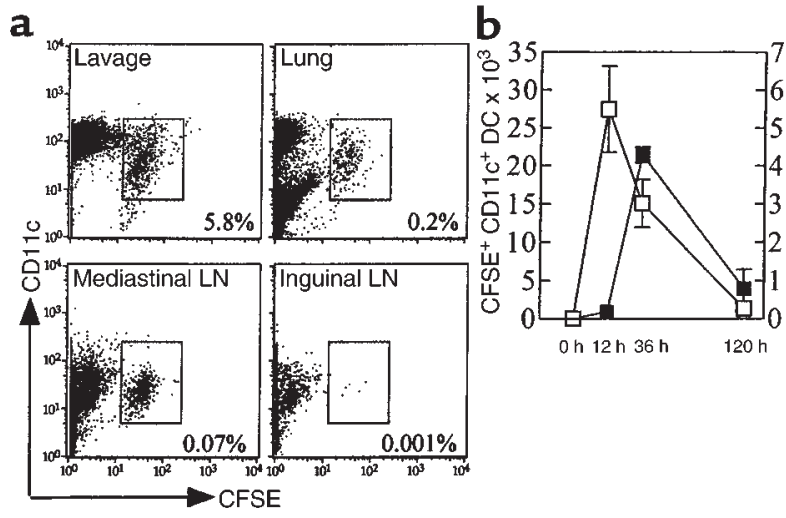

significant increase in total cell counts, mononuclear cells, eosinophils, neutrophils, and lymphocytes in mice that were immunized with $1 \times 10^{6}$ OVA-DCs, compared with animals immunized with PBS-DC (Figure 2a; $P<0.05$ ). Aerosolization with PBS in OVADC-immunized mice did not induce significant changes in BALF compared with PBS-DC/PBSexposed animals or PBS-DC/OVA-exposed animals. Immunization with $1 \times 10^{5}$ OVA-DC and subsequent exposure to OVA aerosol induced an increase in BALF lymphocytes only, whereas immunization with $1 \times 10^{4}$ OVA-DC induced no significant response (data not shown). All subsequent immunizations were therefore performed using $1 \times 10^{6}$ cells.

As studied by flow cytometry and shown in Figure $2 b$, exposure to OVA aerosol in OVA-DC mice induced a significant increase in both $\mathrm{CD}^{+}$and $\mathrm{CD}^{+} \mathrm{T}$ cells

asurement of serum OVA-IgE. Blood was drawn cardiac puncture for measurement of serum OVA-IgE by isotype-specific ELISA (5).

Statistical analysis. Comparison of means between different groups was performed with the MannWhitney $U$ test for unpaired data using the Spreadware Statistics (Spreadware, Palm Desert, California, USA) statistical package. Differences were considered significant if $P<0.05$.

\section{Results}

Injected DCs home to the mediastinal LNs. At various time points after intratracheal injection, we studied the distribution of CFSE-labeled DCs. By 12 hours after instillation, $\mathrm{CFSE}^{+} \mathrm{DCs}$ could be detected in BALF $(14.10 \pm$ $2.99 \%$ of cells), digested lung tissue $(0.17 \pm 0.04 \%$ of cells), and draining mediastinal LNs (0.012 $\pm 0.005 \%)$. No CFSE ${ }^{+}$DCs could be detected in inguinal LNs. As shown in Figure 1a, by 36 hours DCs had further migrated to the mediastinal LNs $(0.07 \pm 0.01 \%)$ and gradually disappeared from the BALF $(5.83 \pm 0.88 \%)$. The number and kinetics of DC migrating into the draining LNs are shown in Figure 1b. Although by 120 hours, a majority of injected cells had disappeared, some $\mathrm{CFSE}^{+}$DCs could still be detected in all compartments $(1.34 \pm 0.15 \%$ of BAL; $0.04 \pm 0.01 \%$ for lung; and $0.016 \pm 0.006 \%$ for $\mathrm{LN})$.

Effect of aerosol exposure on airway inflammation in animals immunized with DCs. Analysis on BALF cells revealed that seven OVA aerosol exposures induced a
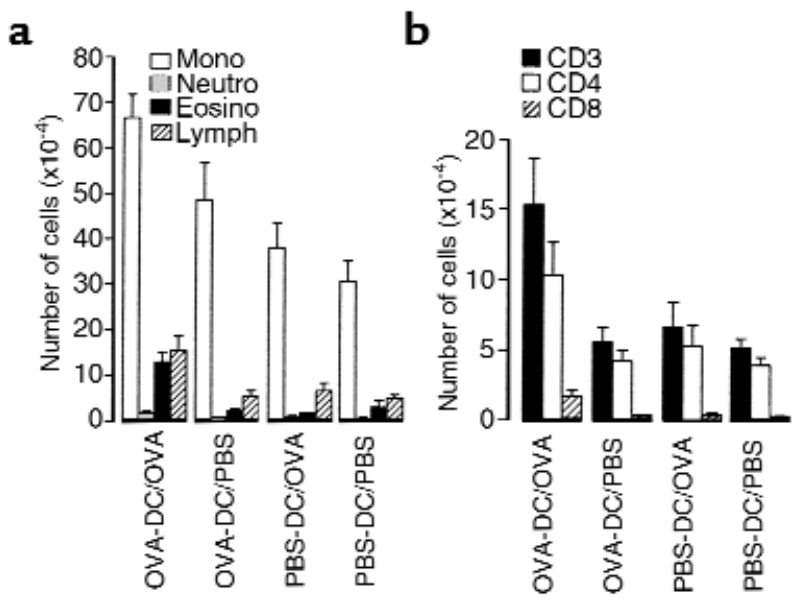

Figure 2

Effect of OVA exposure on the cellular composition of BALF in animals immunized with DCs. On day 0 , mice were immunized by intratracheal administration of $1 \times 10^{6}$ OVA-DCs or control PBS-DCs. On days $14-20$, mice were exposed to 30 minutes of daily OVA or PBS aerosol. At 24 hours after the last aerosol (day 21), BAL was performed. Groups are coded as immunization/aerosol exposure. (a) Differential cell counts based on Giemsa staining. (b) Total T lymphocytes (CD3) and subsets (CD4 and CD8) in BALF as determined by flow cytometry. Results are expressed as means \pm SEM from eight to ten mice per group. Mono, monocytes; neutro, neutrophils; eosino, eosinophils; lymph, lymphocytes. 

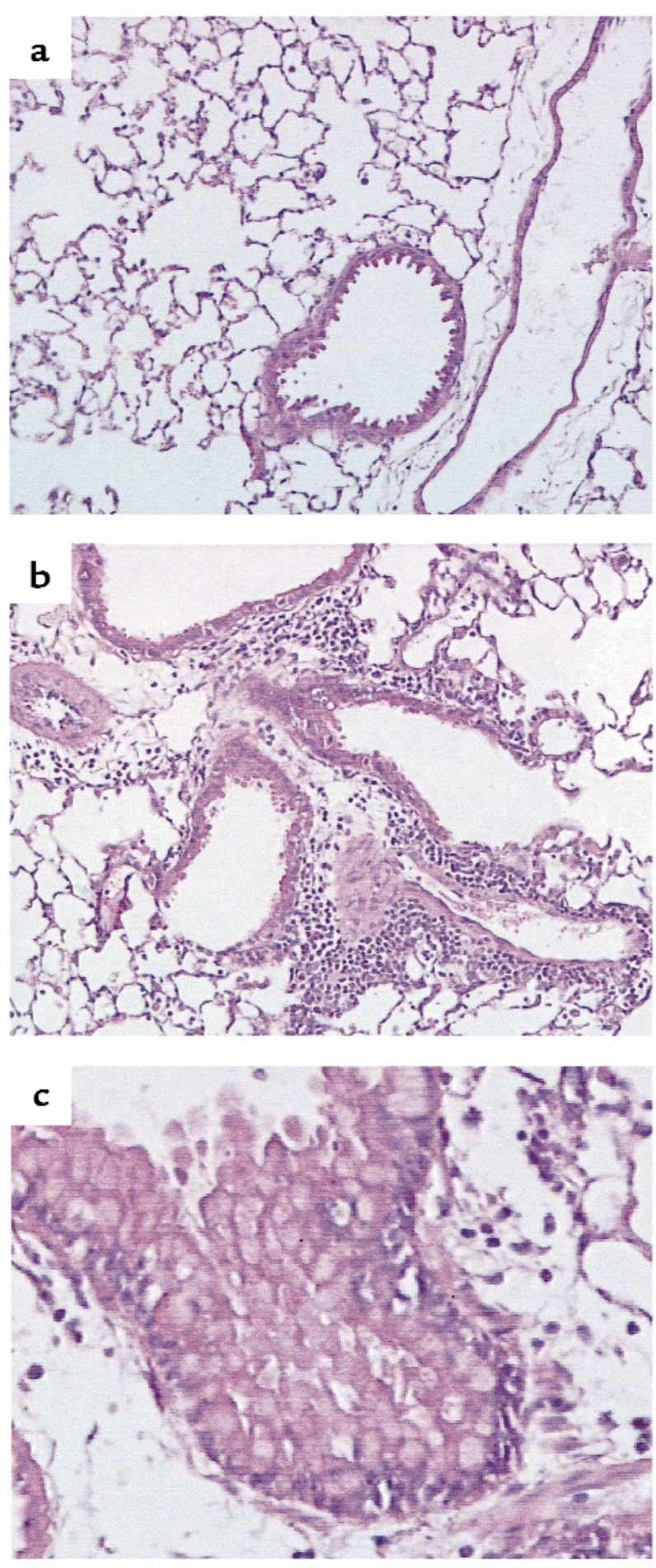

Figure 3

Effect of OVA exposure on lung histology in animals immunized with DCs. Mice were treated as in Figure 2. At 24 hours after the last aerosol, lungs were fixed and processed for histological analysis. H\&E staining. $\times 400$. (a) Representative section from a mouse immunized with PBS-DC and exposed to PBS aerosol. No abnormalities are noticed. ( $\mathbf{b}$ and $\mathbf{c}$ ) Representative sections from mice immunized with OVA-DC and subsequently exposed to OVAaerosol. These mice develop peribronchial and perivascular eosinophil-rich infiltrates (b) and airway mucosal changes typical of goblet cell hyperplasia (c). compared with PBS exposure. In the absence of secondary OVA exposure, there was no difference in total $\mathrm{CD}^{+} \mathrm{T}$-cells or T-cell subsets in animals immunized with OVA-DC compared with those immunized with PBS-DC. The early activation marker CD69 was expressed on $62.9 \pm 3.2 \%$ of $\mathrm{CD} 4^{+} \mathrm{T}$ cells, and the late activation marker CD25 (IL-2R) was expressed on 34.0 $\pm 3.5 \%$ of $\mathrm{CD}^{+} \mathrm{T}$ cells in the OVA-DC/OVA group compared with $11.5 \pm 0.9 \%$ and $5.4 \pm 0.8 \%$, respectively, in the PBS-DC/PBS group $(P<0.05)$.

Lung sections from OVA-DC mice exposed to PBS aerosol (data not shown) or PBS-DC mice exposed to OVA (Figure 3a) or PBS aerosol demonstrated no pathological changes. In contrast, histological analysis of lung tissue from OVA-DC/OVA-exposed mice revealed intense multifocal eosinophilic lesions localized mainly in the peribronchial submucosa, but also marked in the perivascular area (Figure $3 \mathrm{~b}$ ). The vascular wall was grossly edematous, and in some inflamed blood vessels, eosinophils were seen to adhere to vascular endothelium. Epithelial changes of goblet cell hyperplasia were readily seen (Figure 3c).

Effect of aerosol exposure on cytokine levels in animals immunized with DCs. The type of Th response being induced by DCs was determined. As shown in Figure 4a, OVA exposure in OVA-DC mice induced a specific twofold increase in the amount of IL-4 detected in BALF, compared with that detected in PBS-DC/PBS mice $(P<0.05)$. There was no difference in the amount of IFN- $\gamma$ detectable in BALF. Levels of IL-5 were below the detection limit of our assay.

In vitro restimulation of $\mathrm{LN}$ cells (Figure 4, b and c) with OVA demonstrated that mediastinal lymphocytes from OVA-DC/OVA mice produced 27 times more IL$4(747.4 \pm 16.9$ vs. $27.2 \pm 0.1 \mathrm{pg} / \mathrm{mL} ; P<0.05), 1.8$ times more IL-5 (3,120.2 \pm 13.3 vs. $1,698.6 \pm 48.6 \mathrm{pg} / \mathrm{mL} ; P<$ $0.05)$, and equal amounts of IFN- $\gamma(580 \pm 63.6$ vs. 876.6 $\pm 172 \mathrm{pg} / \mathrm{mL} ; P>0.05$ ) compared with those from PBS-DC/PBS mice. In nondraining LNs, levels of all cytokines were identical between OVA-DC/OVA and PBS-DC/PBS mice.

To investigate whether OVA exposure in OVA-DC mice led to selective infiltration of the lungs with lymphocytes producing either Th1- or Th2-type cytokines, intracellular staining for cytokines was performed on BALF cells (Figure 5). Data on percentage expression on $\mathrm{CD}_{4}^{+}$and $\mathrm{CD}^{+}$cells in OVA-DC/OVA and PBS-DC/PBS mice are summarized in Table 1. Simultaneous staining for IL-5 and IFN- $\gamma$ was not performed in the PBS-DC/PBS group, due to lower cell yield in this group.

To study the functional role of IL-4 in this model, DCs were grown from wild-type donors in the absence of exogenous IL-4, and transferred into $\mathrm{IL}_{-4^{+/+}}$or $\mathrm{IL}_{-4^{-/-}}$ mice. Aerosolization with OVA after immunization with OVA-DC induced airway eosinophilia in $\mathrm{IL}_{-4}^{+/+}$ mice $\left(47.7 \pm 4.75 \%\right.$ of BALF cells) but not in $\mathrm{IL}^{-4^{-/-}}$mice $(0.2 \pm 0.14 \%$ of cells). It should be noted that in this experiment, the percentage of BALF eosinophilia in the OVA-DC/OVA group was considerably higher than the 
Table 1

Intracellular staining for cytokines on T cells obtained from BAL

\begin{tabular}{|c|c|c|c|c|c|c|}
\hline \multirow[t]{2}{*}{ Subset } & \multirow[t]{2}{*}{ Group } & \multicolumn{5}{|l|}{ Cytokines $^{\mathrm{A}}$} \\
\hline & & IFN- $\gamma$ & IL-4 & IFN- $\gamma /$ IL-4 & IL-5 & IFN- $\gamma /$ IL5 \\
\hline CD4 & $\begin{array}{l}\text { OVA-DC/OVA } \\
\text { PBS-DC/PBS }\end{array}$ & $\begin{aligned} 11.9 & \pm 1.3 \\
6.7 & \pm 1.6^{B}\end{aligned}$ & $\begin{array}{l}17.1 \pm 3.4 \\
11.9 \pm 2.7^{B}\end{array}$ & $\begin{array}{l}6.8 \pm 1.1 \\
2.1 \pm 0.4^{B}\end{array}$ & $\begin{array}{c}10.4 \pm 2.6 \\
-\end{array}$ & $\begin{array}{c}3.4 \pm 0.6 \\
-\end{array}$ \\
\hline CD8 & $\begin{array}{l}\text { OVA-DC/OVA } \\
\text { PBS-DC/PBS }\end{array}$ & $\begin{array}{l}27.1 \pm 1.4 \\
14.4 \pm 2.8^{\mathrm{B}}\end{array}$ & $\begin{array}{l}1.8 \pm 0.2 \\
2.1 \pm 0.4\end{array}$ & $\begin{array}{l}0.9 \pm 0.1 \\
0.4 \pm 0.1\end{array}$ & $\begin{array}{c}0.4 \pm 0.1 \\
-\end{array}$ & $\begin{array}{l}0.1 \pm 0.1 \\
-\end{array}$ \\
\hline
\end{tabular}

A Cells were obtained from BAL after 7 days of OVA or PBS aerosol and restimulated in vitro for 5 hours using anti-CD3 and anti-CD28 antibodies in the presence of monensin. Shown is the mean percentage of cells expressing a particular cytokine or combination of cytokines \pm SEM from eight mice per group. B $P<0.05$ compared with OVA-DC/OVA group.

$13.6 \pm 1.9 \%$ in our initial experiments reported in Figure $2 \mathrm{a}$. This can be explained by a prolongation of the OVA-pulsing period, which was initially 2 hours before adoptive transfer and was subsequently prolonged to an overnight pulsing in our later experiments (see Methods). The analysis of BALF of wild-type mice immunized with OVA-DC obtained from $\mathrm{IL4}^{-/-}$or $I L 4^{+/+}$donors revealed a similar degree of airway eosinophilia in both groups (data not shown).

Role of signaling through T1/ST2. T1/ST2 is a surface marker known to be differentially expressed on Th2 cells $(16,19,20)$. The expression of T1/ST2 on $\mathrm{CD}^{+} \mathrm{T}$ cells in BALF and in mediastinal and inguinal LNs was investigated after seven aerosol exposures in OVA-DC or PBS-DC mice. The frequency of T1/ST2-expressing $\mathrm{CD}^{+}$cells was highest in BALF $(62.0 \pm 3.8 \%$ in OVADC/OVA vs. $8.4 \pm 1.5 \%$ in PBS-DC/PBS mice (Figure 6a). This decreased to $13.6 \%$ of $\mathrm{CD}^{+}{ }^{+} \mathrm{T}$ cells in the mediastinal LNs of OVA-DC/OVA mice (versus 3.5\% in PBS$\mathrm{DC} / \mathrm{PBS}$ mice). In nondraining inguinal LNs, the expression of T1/ST2 was the lowest and similar in both groups (2.9\% vs. $2.7 \%$ respectively). T1/ST2 was only weakly expressed on $\mathrm{CD}^{+} \mathrm{CD}^{-} \mathrm{T}$ cells (i.e., $\mathrm{CD}^{+} \mathrm{T}$ cells). We next determined the expression of a putative ligand for T1/ST2 on DC grown from our bone marrow cultures. As the ligand for murine T1/ST2 has not been cloned, no antibodies that directly stain the T1/ST2 ligand are available. We have previously cloned the extracellular domain of T1/ST2 into a vector containing the human IgG1 constant region, generating an expressed soluble T1/ST2-Ig fusion protein (16). As seen in Figure 6b, DCs bound T1/ST2-Ig weakly but consistently, suggesting the expression of a ligand for T1/ST2.

We investigated the functional contribution of T1/ST2 to the establishment of eosinophilic airway inflammation by giving either anti-T1/ST2 mAb (3E10) or T1/ST2-Ig during the period of intratracheal injection of DCs. Control animals received either rat IgG or hu-Ig. The intratracheal injection of either 3E10 (Figure 7a) or T1/ST2-Ig (Figure 7b) at the time of sensitization by OVA-DC blocked the development of OVA-induced airway eosinophilia. Moreover, total numbers of monocytes, neutrophils, and lymphocytes were significantly reduced. These changes were also apparent on histological analysis (data not shown).
Role of costimulation through CD28 on T cells. The development of Th2 responses has been shown to depend on strong costimulation provided by CD80/CD86 on APCs to CD28 on T cells (21). DCs were purified from $C D 28^{+/+}$ donors and pulsed overnight with $100 \mu \mathrm{g} / \mathrm{mL}$ of OVA. As expected, immunization with $0.5 \times 10^{6}$ OVA-DC and subsequent exposure to OVA aerosol induced an eosinophilic response in the airways of $C D 28^{+/+}$mice $\left(32.2 \pm 17.7 \times 10^{4}\right.$ eosinophils/lavage) (Figure 8a). However, this eosinophilic response was completely suppressed in CD28 $8^{-/-}$mice $\left(1.13 \pm 1.1 \times 10^{4}\right.$ eosinophils/lavage). The absence of eosinophilia in $\mathrm{CD} 28^{-/-}$mice was also apparent from histological analysis (data not shown). Despite these dramatic differences, the number of $\mathrm{CD}^{+} \mathrm{T}$ cells in BALF was identical between groups $\left(0.6 \pm 0.1 \times 10^{5}\right.$ in $\mathrm{CD} 28^{+/+}$versus $0.4 \pm 0.2 \times 10^{5}$ in $\left.C D 28^{-/-}\right)$. The number of $\mathrm{CD}^{+}$cells expressing CD25 and T1/ST2 was, however, markedly reduced $(13.8 \pm 1.9 \%$ vs. $4.0 \pm 1.6 \%$ and $14.5 \pm$ $4.6 \%$ vs. $2.0 \pm 0.4 \%$, respectively) in $C D 28^{-/-}$mice. To determine the type of Th response induced in the absence of CD28, we measured cytokine levels after in vitro restimulation with OVA of T cells from draining LNs of OVADC/OVA mice. The levels of IFN- $\gamma$ and IL-4 were significantly lower in $C D 28^{-/-}$mice $(1,050 \pm 67.5 \mathrm{pg} / \mathrm{mL}$ and 32 $\pm 3 \mathrm{pg} / \mathrm{mL}$, respectively) compared with $C D 28^{+/+}$mice
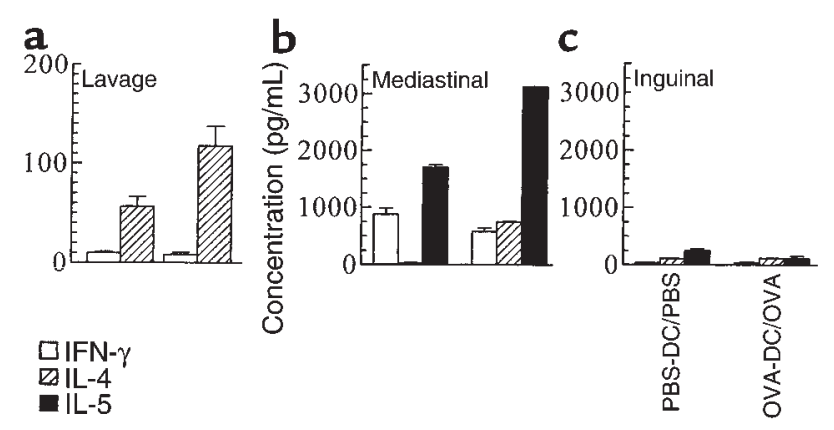

\section{Figure 4}

Measurement of cytokine levels in unconcentrated BALF and after in vitro restimulation of $L N$ cells with specific antigen. Mice were treated as in Figure 2. Groups are coded as immunization/exposure. (a) BALF was taken 24 hours after the last aerosol exposure and assayed for the presence of IL- 4 and IFN- $\gamma$ by ELISA. IL- 5 could not be detected in BALF. (b and c) Mediastinal and inguinal LNs were resected, and single-cell suspensions were cultured in vitro for 96 hours in the presence of OVA. Results represent means \pm SEM from five to eight animals per group. 


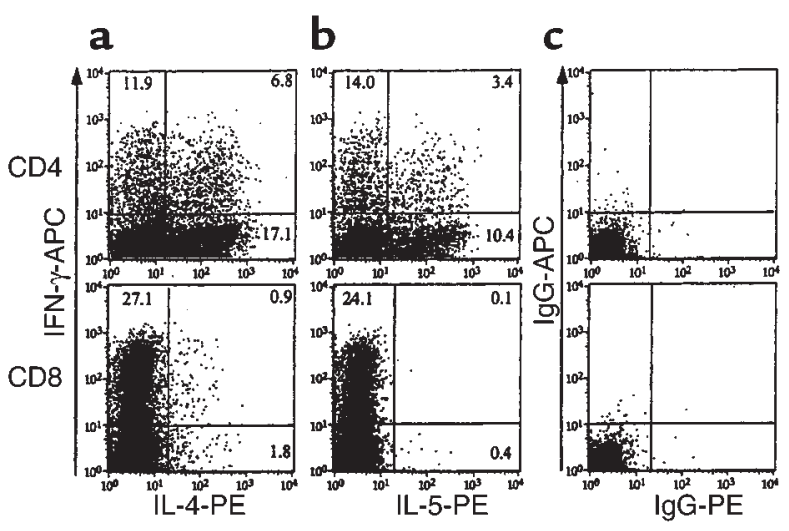

Figure 5

Intracellular detection of IFN- $\gamma$, IL-4, and IL-5 in lymphocytes from BALF of OVA-DC/OVA mice. As described in Methods, cells were stained for IFN- $\gamma$ (a and b), IL-4 (a), IL-5 (b), and isotype controls (c). Dot plots shown were gated on $\mathrm{CD}^{+} \mathrm{CD} 4^{+}$lymphocytes (top) or $\mathrm{CD} 3^{+} \mathrm{CD} 8^{+}$lymphocytes (bottom). Number of cells staining for each cytokine are expressed as a percentage of $\mathrm{CD}^{+}$or $\mathrm{CD} 8^{+}$cells. Representative of all mice in the group $(n=8)$.

$(5,441 \pm 1,742 \mathrm{pg} / \mathrm{mL}$ and $80 \pm 44 \mathrm{pg} / \mathrm{mL}$, respectively. The levels of IL-5 were lower in $C D 28^{-1-}(640 \pm 60 \mathrm{pg} / \mathrm{mL})$ than in $\mathrm{CD} 28^{+/+}$mice $(877 \pm 354 \mathrm{pg} / \mathrm{mL})$, but this did not reach statistical significance. In contrast, intracellular staining for cytokines after polyclonal activation with $\mathrm{PMA} /$ ionomycin revealed that individual $\mathrm{CD} 4^{+} \mathrm{T}$ cells obtained from BALF of OVA-DC/OVA mice produced either IFN- $\gamma$ or IL-4 in $\mathrm{CD} 28^{+/+}$mice and that the percentage expression of IL-4 in individual cells was not affected by knockout of the CD28 gene (Figure 8b). However, the percentage expression of IFN- $\gamma$ in $C D 28^{-/-} \mathrm{T}$ cells was almost twice as high compared with $C D 28^{+/+}$cells.

\section{Discussion}

In an attempt to define the role of DCs in the regulation of lung mucosal immunity, we have injected antigen-pulsed myeloid DCs into the trachea of naive mice. During the afferent phase of the pulmonary immune response, $\mathrm{Ag}$ is deposited into the deeper airways and captured by endogenous APCs, which transport it into the draining LNs $(3,6,22)$. In our system, CFSE-labeled DC could be traced in BALF and draining mediastinal LNs already 12 hours after injection into the trachea, but not in nondraining $\mathrm{LN}$ or spleen (data not shown). By 36 hours after injection, labeled DCs started to disappear from the BAL compartment, consistent with a further migration into the draining LNs. In agreement with data from subcutaneous injection of DCs, the majority of migrated DCs had disappeared from the draining LNs by 120 hours after injection, although significant numbers of cells were retained in all compartments of the lung studied (23). Using the same model of adoptive transfer of DCs into the trachea, we have recently demonstrated that migrated DCs interact with naive $T$ cells in the mediastinal LNs during the so-called central processing phase of the adaptive pulmonary immune response, leading to lymphocyte division and generation of effector cells (6). The time course and distribution of this primary T-cell response are highly reminiscent of the kinetics and direction of migration of DCs, as Tcell division occurs before 48 hours and is restricted to the mediastinal lymph nodes. This restricted migration of DCs into the mediastinal LNs of the lung leads to a compartmentalized immune response (22).

During the effector phase of the immune response, effector $\mathrm{T}$ cells are recruited into the lung and again stimulated by DCs to release cytokines and orchestrate airway inflammation $(5,24)$. The occurrence and the nature of the secondary effector response reflects the character of the primary response and the development of polarized Th1 or Th2 responses during the central processing phase (25). Challenge with aerosolized OVA in mice that were immunized by intratracheal injection of OVA-DC, led to the accumulation of activated $\mathrm{CD} 4^{+}$ $T$ lymphocytes in the airways and to eosinophilic airway inflammation and goblet cell hyperplasia, both characteristic of allergic asthma. As the development of goblet cell hyperplasia and eosinophilic airway inflammation is clearly dependent on IL-4 receptor signaling and local IL-5 production by $\mathrm{CD} 4^{+} \mathrm{T}$ cells, respectively (26), we speculated that a Th2-dominated response was induced by intratracheal injection of DCs. Supporting this hypothesis, we measured increased levels of Th2 but not Th1 cytokines in BALF of OVA-DC/OVA mice compared with PBS-DC/PBS mice. Moreover, when lymphocytes of the draining mediastinal LNs of OVA-DC/OVA mice were restimulated with specific Ag, they similarly produced increased levels of IL4 and IL-5 and identical levels of IFN- $\gamma$ compared with controls. These high levels of Th2 cytokine production were mainly seen in the LNs draining the lung, again suggesting the presence of a very compartmentalized immune response. Intracellular

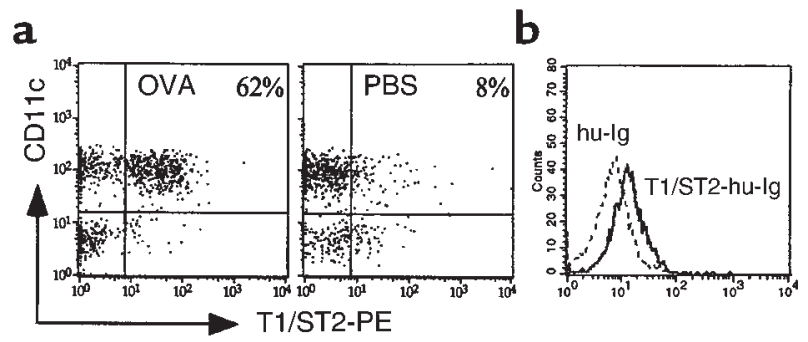

\section{Figure 6}

(a) T1/ST2 expression on BALF cells. Mice were immunized with OVADC and subsequently challenged with OVA as described in Methods. Control mice (PBS) were immunized with PBS-DC and challenged with PBS. Single-cell suspensions were surface stained with mAb's against CD3, CD4, and T1/ST2. Dot plots were gated on CD3+ viable lymphocytes. The percentage of $\mathrm{CD}^{+}$lymphocytes expressing $\mathrm{T} 1 / \mathrm{ST} 2^{+}$is indicated. (b) Expression of a putative ligand for T1/ST2 on mouse DCs. Bone marrow DCs were stained for CD11c, MHC class II, and T1/ST2-Ig, followed by secondary anti-hu-Ig-FITC. As a control, staining was performed using hu-lg as primary antibody (broken line). Histogram was gated on $\mathrm{CD} 11 \mathrm{c}^{+} \mathrm{MHC}$ class $\mathrm{II}^{+}$cells and is representative of three separate experiments. 


\section{Figure 7}

Effect of blocking the interaction ofT1/ST2 with its ligand during sensitization by OVA-DC on the development of eosinophilic inflammation. (a) On day 0 of the experiment, mice received an intratracheal injection of $1 \times 10^{6}$ OVA-DC simultaneously with a rat $\mathrm{mAb}$ against T1/ST2 (3E10) or as a control rat IgG. (b) In another experiment, mice received an intratracheal injection of $1 \times 10^{6}$ OVA-DC simultaneously with a T1/ST2-Ig fusion protein or as a control hu-IgG. Mice were subsequently exposed to OVA aerosol from day 14 to day 20 and BALF recovered 24 hours after the last aerosol challenge.

staining for cytokines on individual BALF $\mathrm{CD}^{+} \mathrm{T}$ lymphocytes revealed that $\mathrm{CD} 4^{+}$lymphocytes were the predominant cellular source of IL-4 and IL-5. However, enhanced numbers of $\mathrm{CD}^{+}$and $\mathrm{CD}^{+}$cells producing only IFN- $\gamma$ were also clearly detected in BALF. This suggests that eosinophilic inflammation in mice is characterized by an expansion of $\mathrm{CD}^{+} \mathrm{Th} 2$ cells, without selectively abrogating Th1 cells. Similarly, in addition to an increased number of IL-4- and IL-5-producing cells, a population of IFN- $\gamma$-producing $\mathrm{CD}^{+} \mathrm{T}$ cells has been demonstrated in BALF from asthmatic subjects (27). A final argument for the induction of a specific Th2 response by DC was the total absence of the eosinophilic response in mice genetically lacking IL-4. By blocking IL4 with antibodies at various time points of the response, we have previously shown that the absence of IL-4 mainly affects the development of Th2 responses during the central processing phase (i.e., sensitization), whereas the effector phase is not affected $(28,29)$.

Despite the high levels of IL-4 measured, we could not detect significant levels of OVA-specific IgE production in the serum of OVA-DC/OVA-exposed mice. This is not surprising, as we immunized the mice by injection of OVA-pulsed DCs, in the absence of free OVA antigen. As such, no free antigen was available for stimulation of naive $B$ cells. Other protocols in which protein-specific Th2-dependent immunoglobulins were detected after injection of DCs included the injection of a booster of soluble protein $(11,30)$.

Despite the known functional dichotomy of murine Th responses into Th1- or Th2-cytokine-secreting subsets, few surface molecules can discriminate between them $(16,20)$. The T1/ST2 orphan receptor was found to be differentially expressed on Th2 clones and on recently differentiated Th2 cells in vitro $(16,20)$. The T1/ST2 belongs to the IL-1 receptor superfamily and does not bind to any of its known ligands (31). It was recently demonstrated that IL-4-, IL-5-, and IL-10-producing $\mathrm{CD}^{+} \mathrm{T}$ cells isolated from granulomas induced by Schistosoma mansoni eggs express T1/ST2 in vivo (19). Similarly, BALF and mediastinal $\mathrm{CD}^{+} \mathrm{T}$ cells expressed T1/ST2 in OVADC/OVA animals, whereas $\mathrm{T}$ cells from a distant site did not. This strongly suggested that T1/ST2 expression in vivo colocalized with the compartmentalized Th2-dominated immune response. In addition to its role as a marker for Th2 cells, we and others have recently described that signaling through T1/ST2 is critical for

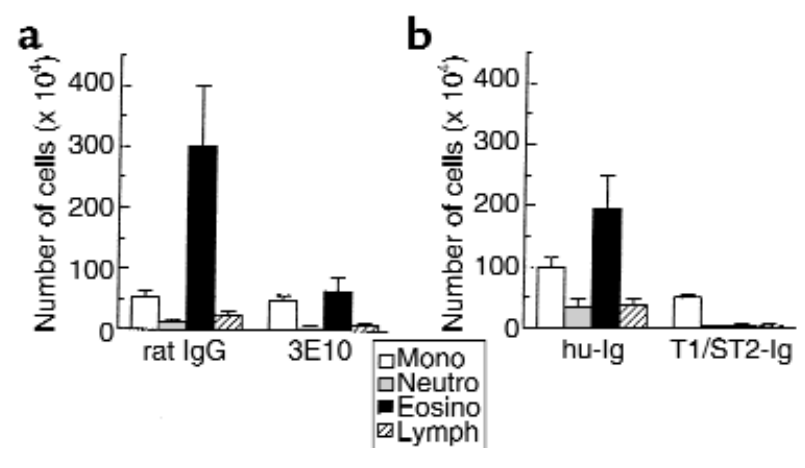

Th2 development and effector function in vitro and in vivo $(16,32,33)$, although some controversy exists as to the exact role of T1/ST2 in Th development (34). To address this further, we analyzed whether DC-induced Th2 development in the airways was inhibited by blocking the interaction of T1/ST2 with its (unknown) ligand. By injecting blocking antibodies or T1/ST2-Ig fusion protein at the time of injection of DCs, the sensitization to inhaled antigen was completely suppressed. This strongly suggested the functional expression of a ligand for T1/ST2 on DCs, as shown by weak binding of the T1/ST2-Ig protein. A putative ligand has also been identified on other APC types such as B cells and follicular DCs (35). Definite molecular proof of expression on these cells, as well as studies on its regulation, will await molecular cloning of the ligand for murine T1/ST2.

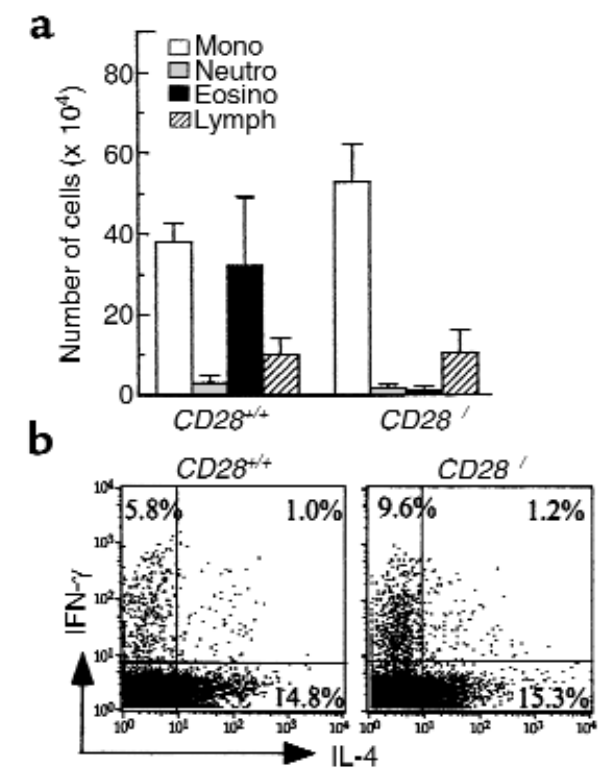

Figure 8

Effect of absence of CD28 on the development of OVA-induced eosinophilic airway inflammation. On day $0, C D 28^{+/+}$and $C D 28^{-/-}$ mice received $5 \times 10^{5}$ OVA-DC from wild-type mice. Mice were subsequently exposed to OVA aerosol from day 14 to day 20 and BALF was recovered 24 hours after the last aerosol challenge. (a) Differential cell counting on BALF. Results represent means \pm SEM from six mice per group. (b) Intracellular staining for cytokines on BALF T cells. Cells were restimulated with $\mathrm{PMA} /$ ionomycin in the presence of monensin and stained for IFN- $\gamma$ and IL-4. Percentage expression of each cytokine on $\mathrm{CD} 3^{+} \mathrm{CD} 4^{+}$cells is indicated. 
The strong expression of costimulatory molecules such as CD80 and CD86 is one of the features discriminating lung DCs from other APCs such as B cells and macrophages (36). It is controversial at present whether signaling through CD28 by either of these ligands affects the potential of $\mathrm{T}$ cells to develop into either Th1 or Th2 cells $(37,38)$. It has been suggested that the induction of Th2 responses in vitro and in vivo depends on the delivery of a strong costimulus by the APC to naive T cells (38). We therefore hypothesized that Th2 development and eosinophilia induced by DC would be absent in mice with a disrupted CD28 gene. Although we found that airway eosinophilia was completely abrogated, the number of BALF $\mathrm{T}$ cells induced by OVA aerosol was identical between $C D 28^{-1-}$ and wild-type mice. By analogy, recent work in a $S$. mansoni-induced model of airway inflammation has shown that CD28 deficiency abolishes airway eosinophilia but does not affect the initial recruitment of T cells into Ag-challenged airways (39). The absence of airway eosinophilia in $\mathrm{CD} 28^{-/-}$mice could be due to defective Th2 priming or to defective generation of effector function in Th2 cells during challenge of the airways. Studies using either CTLA-4Ig or blocking anti-CD80 and CD86 mAb's have indeed shown that costimulation via the CD80/86-CD28 pathway is essential during both the central processing and the effector phases of the immune response leading to airway eosinophilia (39-41). To clarify this point, lung effector $\mathrm{CD}^{+} \mathrm{T}$ cells of $\mathrm{CD} 28^{-/-}$mice were restimulated in a costimulation-independent fashion using PMA/ionomycin and studied for single-cell expression of cytokines. We found a normal capacity of these cells to produce IL-4 with an increased tendency to produce IFN- $\gamma$, indicating that the priming of Th 2 cells by DCs was unaltered in $\mathrm{CD} 28^{--}$mice. However, when LN T cells were restimulated with OVA in a costimulationdependent assay, levels of both Th1 and Th2 cytokines were grossly diminished in $\mathrm{CD} 28^{-/-}$mice. We therefore favor the hypothesis that airway eosinophilia was abolished mainly by a defective activation of effector $T$ cells in the lung of $\mathrm{CD} 28^{-/-}$mice. Consistent with this, we observed a reduced expression of the activation marker $\mathrm{CD} 25$ on $\mathrm{CD}_{4}^{+} \mathrm{T}$ cells in $\mathrm{CD} 28^{-/-}$mice.

In conclusion, DCs induce Th2-dominated sensitization to inhaled Ag, leading to eosinophilic airway inflammation. These results and our findings that DCs are essential for the maintenance of airway eosinophilia suggest that these cells are critical to the pathogenesis of asthma $(5,24)$. Therefore, interacting with DC function is a therapeutic option for this disease.

\section{Acknowledgments}

This work was supported by the Concerted Research Initiative of the University of Ghent (G.O.A. 98-6) and by the Fund for Scientific Research Vlaanderen (G.0393.99). We gratefully acknowledge the technical assistance of G. Barbier, K. De Saedeleer, M. Gosselin, and S. Lin. The
Inflammation Division of Millenium Pharmaceuticals is supported by Astra Pharmaceuticals.

1. Robinson, D.R., et al. 1992. Predominant Th2-like bronchoalveolar T lymphocyte population in atopic asthma. N. Engl. J. Med. 326:298-304.

2. Banchereau, J., and Steinman, R.M. 1998. Dendritic cells and the control of immunity. Nature. 392:245-252.

3. Holt, P.G., Schon-Hegrad, M.A., and Oliver, J. 1988. MHC class II antigen-bearing dendritic cells in pulmonary tissues of the rat (regulation of antigen presentation activity by endogenous macrophage populations). J. Exp. Med. 167:262-274.

4. Schon-Hegrad, M.A., Oliver, J., McMenamin, P.G., and Holt, P.G. 1991. Studies on the density, distribution and surface phenotype of intraepithelial class II major histocompatibility complex antigen (Ia)-bearing dendritic cells (DC) in the conducting airways. J. Exp. Med. 173:1345-1356.

5. Lambrecht, B.N., Salomon, B., Klatzmann, D., and Pauwels, R.A. 1998. Dendritic cells are required for the development of chronic eosinophilic airway inflammation in response to inhaled antigen in sensitized mice. J. Immunol. 160:4090-4097.

6. Lambrecht, B.N., Pauwels, R.A., and Fazekas de St.Groth, B. 2000. Induction of rapid $\mathrm{T}$ cell activation, division and recirculation by intratracheal injection of dendritic cells in a TCR-transgenic model. J. Immunol. 164:2937-2946.

7. Kalinski, P., Schuitemaker, J.H., Hilkens, C.M., Wierenga, E.A., and Kapsenberg, M.L. 1999. Final maturation of dendritic cells is associated with impaired responsiveness to IFN-gamma and to bacterial IL12 inducers: decreased ability of mature dendritic cells to produce IL12 during the interaction with Th cells. J. Immunol. 162:3231-3236.

8. Maldonado-Lopez, R., et al. 1999. CD8alpha+ and CD8alpha- subclasses of dendritic cells direct the development of distinct $T$ helper cells in vivo. J. Exp. Med. 189:587-592.

9. Rissoan, M.C., et al. 1999. Reciprocal control of T helper cell and dendritic cell differentiation. Science. 283:1183-1186.

10. Macatonia, S.E., et al. 1995. Dendritic cells produce IL-12 and direct the development of Th1 cells from naive CD4(+) T cells. J. Immunol. 154:5071-5079.

11. Stumbles, P.A., et al. 1998. Resting respiratory tract dendritic cells preferentially stimulate $\mathrm{T}$ helper cell type 2 (Th2) responses and require obligatory cytokine signals for induction of Th 1 immunity. $J$. Exp. Med. 188:2019-2031.

12. De Becker, G., et al. 1998. Regulation of T helper cell differentiation in vivo by soluble and membrane proteins provided by antigen-presenting cells. Eur. J. Immunol. 28:3161-3171.

13. De Veerman, M., et al. 1999. Retrovirally transduced bone marrowderived dendritic cells require cognate CD4+ T cell help to elicit protective and therapeutic antitumor immunity. J. Immunol. 162:144-151.

14. Lambrecht, B.N., et al. 1999. Endogenously produced substance P contributes to lymphocyte proliferation induced by dendritic cells and direct TCR ligation. Eur. J. Immunol. 29:3815-3825.

15. Ho, W., and Furst, A. 1973. Intratracheal instillation method for mouse lungs. Oncology. 27:385-393.

16. Lohning, M., et al. 1998. T1/ST2 is preferentially expressed on murine Th2 cells, independent of interleukin 4, interleukin 5, and interleukin 10, and important for Th2 effector function. Proc. Natl. Acad. Sci. USA. 95:6930-6935.

17. Harding, F.A., McArthur, J.G., Gross, J.A., Raulet, D.H., and Allison, J.P. 1992. CD28-mediated signalling co-stimulates murine T cells and prevents induction of anergy in T-cell clones. Nature. 356:607-609.

18. Van Oosterhout, A.J., et al. 1998. Allergen immunotherapy inhibits airway eosinophilia and hyperresponsiveness associated with decreased IL-4 production by lymphocytes in a murine model of allergic asthma. Am. J. Respir. Cell Mol. Biol. 19:622-628.

19. Lohning, M., et al. 1999. T1/ST2 expression is enhanced on CD4+ T cells from schistosome egg-induced granulomas: analysis of Th cell cytokine coexpression ex vivo. J. Immunol. 162:3882-3889.

20. Xu, D., et al. 1998. Selective expression of a stable cell surface molecule on type 2 but not type 1 helper T cells. J. Exp. Med. 187:787-794.

21. Bluestone, J.A. 1995. New perspectives of CD28-B7-mediated T cell costimulation. Immunity. 2:555-559.

22. Xia, W.J., Pinto, C.E., and Kradin, R.L. 1995. The antigen-presenting activities of Ia+ dendritic cells shift dynamically from lung to lymph node after an airway challenge with soluble antigen. J. Exp. Med. 181:1275-1283.

23. Hermans, I.F., Ritchie, D.S., Yang, J., Roberts, J.M., and Ronchese, F. 2000. CD8+ T cell-dependent elimination of dendritic cells in vivo limits the induction of antitumor immunity. J. Immunol. 164:3095-3101.

24. Lambrecht, B.N., Carro-Muino, I., Vermaelen, K., and Pauwels, R.A. 1999. Allergen-induced changes in bone-marrow progenitor and airway dendritic cells in sensitized rats. Am. J. Respir. Cell Mol. Biol. 20:1165-1174. 
25. Lambrecht, B.N., Peleman, R.A., Bullock, G.R., and Pauwels, R.A. 2000. Sensitization to inhaled antigen by intratracheal instillation of dendritic cells. Clin. Exp. Allergy. 30:214-224.

26. Hogan, S.P., Koskinen, A., Matthaei, K.I., Young, I.G., and Foster, P.S. 1998. Interleukin-5-producing CD4 T cells play a pivotal role in aeroallergen-induced eosinophilia, bronchial hyperreactivity, and lung damage in mice. Am. J. Respir. Crit. Care Med. 157:210-218.

27. Krug, N., et al. 1996. T-cell cytokine profile evaluated at the single cell level in BAL and blood in allergic asthma. Am. J. Respir. Cell Mol. Biol. 14:319-326

28. Brusselle, G.G., Kips, J.C., Joos, G.F., Bluethmann, H., and Pauwels, R.A. 1995. Allergen-induced airway inflammation and bronchial responsiveness in wild-type and interleukin-4-deficient mice. Am.J. Respir. Cell Mol. Biol. 12:254-259.

29. Coyle, A.J., et al. 1995. Interleukin-4 is required for the induction of lung Th2 mucosal immunity. Am. J. Respir. Cell Mol. Biol. 13:54-59.

30. Sornasse, T., et al. 1992. Antigen pulsed dendritic cells can efficiently induce an antibody response in vivo. J. Exp. Med. 175:15-21.

31. Mitcham, J.L., et al. 1996. T1/ST2 signaling establishes it as a member of an expanding interleukin-1 receptor family. J. Biol. Chem. 271:5777-5783.

32. Coyle, A.J., et al. 1999. Crucial role of the interleukin 1 receptor family member T1/ST2 in T helper cell type 2 -mediated lung mucosal immune responses. J. Exp. Med. 190:895-902.

33. Townsend, M.J., Fallon, P.G., Matthews, D.J., Jolin, H.E., and McKen- zie, A.N. 2000. T1/ST2-deficient mice demonstrate the importance of T1/ST2 in developing primary T helper cell type 2 responses. J. Exp. Med. 191:1069-1076.

34. Hoshino, K., et al. 1999. The absence of interleukin 1 receptor-related T1/ST2 does not affect T helper cell type 2 development and its effector function. J. Exp. Med. 190:1541-1548.

35. Gayle, M.A., et al. 1996. Cloning of a putative ligand for the T1/ST2 receptor. J. Biol. Chem. 271:5784-5789.

36. Masten, B.J., and Lipscomb, M.F. 1999. Comparison of lung dendritic cells and B cells in stimulating naive antigen-specific T cells. $J$. Immunol. 162:1310-1317.

37. Brown, D.R., et al. 1996. Limited role of CD28-mediated signals in Thelper subset differentiation. J. Exp. Med. 184:803-810.

38. Rulifson, I.C., Sperling, A., Fields, P.E., Fitch, F.W., and Bluestone, J.A. 1997. CD28 costimulation promotes the production of Th2 cytokines. J. Immunol. 158:658-665.

39. Mathur, M., et al. 1999. CD28 interactions with either CD80 or CD86 are sufficient to induce allergic airway inflammation in mice. Am.J. Respir. Cell Mol. Biol. 21:498-509.

40. Harris, N., et al. 1997. CD80 costimulation is essential for the induction of airway eosinophilia. J. Exp. Med. 185:177-182.

41. Tsuyuki, S., Tsuyuki, J., Einsle, K., Kopf, M., and Coyle, A.J. 1997. Costimulation through B7-2 (CD86) is required for the induction of a lung mucosal $\mathrm{T}$ helper cell 2 (TH2) immune response and altered airway responsiveness. J. Exp. Med. 185:1671-1679. 